\title{
Room temperature study of a strain-induced electronic superstructure on a magnetite (111) surface
}

\author{
N. Berdunov, S. Murphy, G. Mariotto, and I. V. Shvets \\ SFI Nanoscience Laboratory, Physics Department, Trinity College, Dublin 2, Ireland \\ (Received 21 May 2003; revised manuscript received 15 April 2004; published 6 August 2004)
}

\begin{abstract}
A magnetite $\left(\mathrm{Fe}_{3} \mathrm{O}_{4}\right)$ single-crystal (111) surface has been studied at various oxygen-iron surface stoichiometries. The stoichiometry was modified by controlling the in situ sample anneal conditions. We have found the conditions that lead to the formation of an oxygen-rich surface that forms a quasihexagonal superstructure with a $42 \AA$ periodicity. The superstructure is highly regular and was observed by both low-energy election diffraction and scanning tunneling microscopy. The superstructure consists of three regions, two of which have identical atomic scale structures with a periodicity of $2.8 \AA$, and a third having a periodicity that is about $10 \%$ larger $(3.1 \AA)$. The subtle difference in the atomic periodicities between the three areas results from the modulation of intrinsic strain developed along the surface. The superstructure results from electronic effects rather than being a mosaic of different iron oxide terminations. The onset of the superstructure is sensitive to the surface stoichiometry. From our results we could estimate the critical density of defects leading to the disappearance of the superstructure. We have modeled the experimental results and calculated the electron density using density functional theory calculations. The model clearly shows the development of strain along the surface.
\end{abstract}

DOI: $10.1103 /$ PhysRevB.70.085404

PACS number(s): 73.20.At, 71.38.-k, 75.30.-m, 72.80.Ga

\section{INTRODUCTION}

Magnetite is a well-known example of a material that undergoes a metal-insulator transition, one of the most important phenomena in solid-state physics. Magnetite has the inverse spinel crystal structure based on an fcc lattice of oxygen $\left(\mathrm{O}^{2-}\right)$ anions, containing $\mathrm{Fe}$ cations in tetrahedrally (so-called $A$ sites) and octahedrally ( $B$ sites) coordinated interstices (Fig. 1). The $A$ sites are occupied by $\mathrm{Fe}^{3+}$ ions and the $B$ sites by $\mathrm{Fe}^{2+}$ and $\mathrm{Fe}^{3+}$ ions. ${ }^{1}$ At the Verwey temperature $T_{v} \sim 120 \mathrm{~K}$, conduction electrons freeze at the octahedrally coordinated iron sites, forming a charge-ordered state. The exact arrangement of the $\mathrm{Fe}^{2+}$ ions within the lattice below $T_{v}$, and whether short-range or long-range order is present is the subject of a continuing debate. It has even been suggested that the transition may be caused by the dimerization of electron orbital bonds rather than charge ordering. ${ }^{2}$

The surface of magnetite has been studied intensively over the past decade, as it is a highly interesting material for spin electronics. It is one of the few materials with a very high degree of spin polarization at the Fermi level. It is even possible that magnetite is a half-metallic ferromagnet, meaning that it is an insulator in one of the two spin bands and a conductor in the other. Spin electronics almost invariably involves electron transport across or along a material interface. Therefore, an understanding of the structure and elec- a

$\mathrm{Fe}_{3} \mathrm{O}_{4}[111]$ slab

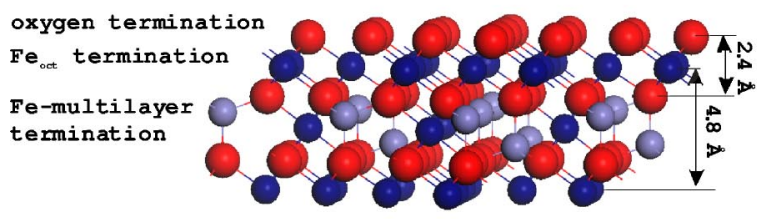

d

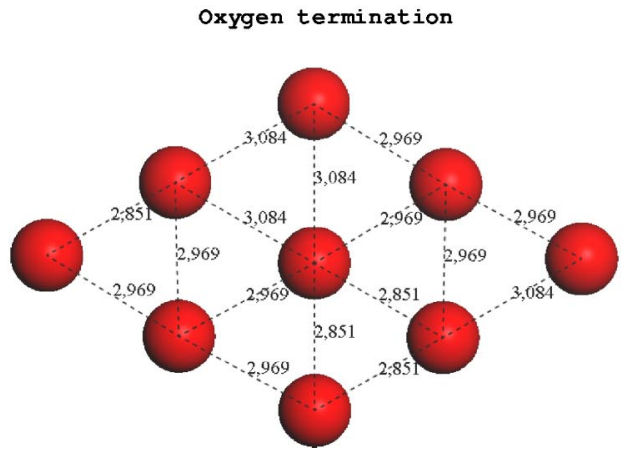

b

Fe-octahedral termination

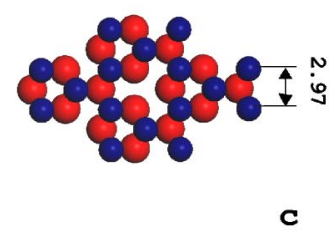

Fe-tetrahedral termination

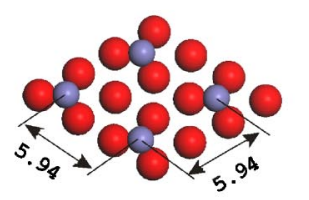

$-\mathrm{O}^{2-}$

$-\mathrm{Fe}_{\mathrm{oct}}^{2+, 3+}($ B-site)
FIG. 1. (Color online) Magnetite [111] slab (a); two different bulk terminations that contain $\mathrm{Fe}$ cations $(b, c)$; and oxygen termination (d). In (b) and (c), oxygen layers shown are positioned below the surface termination. Figure (d) has enlarged scale. 
tronic properties of surfaces/interfaces is a key issue for making progress in this area. At the same time, progress into exploring the potential of magnetite for spin electronics is slowed by the complexity of the magnetite structure. Magnetite has six possible bulk terminations in the [111] direction, ${ }^{3}$ but only two of them have been clearly resolved experimentally. ${ }^{4-6}$ The surface is highly sensitive to the preparation conditions. Moreover, self-consistent band structure calculations of magnetite surfaces and surface reconstruction models for low index terminations are still not available.

Apart from possible transformations associated with the rearrangement of atoms at the surface that should be common for the vast family of spinel oxides, magnetite has an additional unique complexity caused by possible charge rearrangement at the cationic sites. At room temperature the charge ordering has been observed in the bulk. ${ }^{7}$ It has also been reported that under the correct preparation conditions, the magnetite (100) surface may undergo a metal-insulator transition at a temperature above room temperature. ${ }^{8,9}$ It is now clear that the charge order on the (100) surface critically depends on the surface stoichiometry.

Charge ordering on other low index terminations of magnetite has not been studied. However, one can expect that this should also be sensitive to surface stoichiometry. In this paper, we focus on the structural transformations of a $\mathrm{Fe}_{3} \mathrm{O}_{4}$ (111) single-crystal surface and their impact on the electron structure and charge ordering at the surface and in the subsurface layer.

\section{EXPERIMENT}

The ultrahigh vacuum (UHV) experimental setup includes a room-temperature scanning tunneling microscope (STM), a reverse view four-grid low-energy electron diffraction (LEED) optics, and a cylindrical mirror analyzer for Auger electron spectroscopy (AES). The experimental setup is described in detail in Ref. 10.

The magnetite sample was grown by the floating zone technique ${ }^{11}$ and cut along the (111) plane with a precision of $\pm 1^{\circ}$. A characterization of the sample by $x$-ray powder diffractometry and single-crystal high-resolution diffractometry using a Cu target $(\lambda=1.54 \AA)$ showed good agreement with the reference peaks for magnetite. By means of highresolution x-ray diffractometry, we have also verified that the sample miscut was consistent with the expected precision of $\pm 1^{\circ}$. A Verwey transition temperature of $120 \mathrm{~K}$ was measured from the discontinuity in the resistivity versus temperature curve, indicating that the crystal is stoichiometric. Measurements of the magnetization of the sample around $T_{v}$ were performed in fields of 5 to $50 \mathrm{mT}$, using a vibrating sample magnetometer. The value of saturation magnetization of $94 \mathrm{emu} / \mathrm{g}$ obtained from the magnetization measurements at $297 \mathrm{~K}$ is in good agreement with the expected value for magnetite. The values of $T_{v}$ obtained from the magnetization and resistivity measurements are consistent with stoichiometric magnetite. By characterizing the sample using Raman spectroscopy measurements, we were able to positively exclude the presence of other iron oxide phases in the crystal.
The crystal was mechanically polished with diamond paste down to a grain size of $0.05 \mu \mathrm{m}$, cleaned using organic solvents and transferred into the UHV system. The invacuum sample cleaning procedure consisted of repeated cycles of $\mathrm{Ar}^{+}$ion etching (1.0-0.5 keV, 5-15 min) and annealing in UHV at a temperature of $950 \mathrm{~K}$ for a few hours. After a few such cycles, the sample reveals a contaminantfree surface, i.e., AES measurements indicate that the concentration of contaminants is below $1 \%$ and LEED patterns taken on the surface correspond to an unreconstructed bulk termination. The final in situ surface preparation is crucially important to the results presented in this paper, and therefore is described in detail separately for each case.

\section{RESULTS AND DISCUSSION}

The crystal structure of magnetite allows for six ideal bulk terminations of the (111) surface (Fig. 1). Two of them represent terminations consisting of close-packed oxygen layers and the other four are surface planes containing cations $\left(\mathrm{Fe}^{2+}, \mathrm{Fe}^{3+}\right)$. There are significant differences between the four Fe terminations. One of them consists of Fe tetrahedral sites arranged in a hexagonal (honeycomb) lattice with a $6 \AA$ periodicity [Fig. 1(c)]. Another one has octahedral site Fe atoms arranged in a "Kagome" lattice [Fig. 1(b)] with $3 \AA$ interatomic distances. The two remaining Fe-containing terminations are multilayer terminations with octahedral and tetrahedral iron layers separated by $0.6 \AA$ in the [111] direction. One of the two oxygen terminations appears as an oxygen monolayer on top of octahedral $\mathrm{Fe}$, while the other one covers a multilayer of tetrahedral and octahedral $\mathrm{Fe}$ atoms. The atomic periodicities of both oxygen terminations are identical [Fig. 1(d)]. The bulk separation between nearestneighbor anions in these terminations varies between 2.851 and $3.084 \AA$.

All STM, AES, and LEED measurements presented in this article were performed at room temperature. Typically the STM was operated in the constant-current mode. In our study of the magnetite (111) surface we employ tips that we have fabricated from a variety of materials: ${ }^{12}$ nonmagnetic tungsten tips, ferromagnetic Ni tips, and also tips made of antiferromagnetic MnNi alloy. Antiferromagnetic tips are interesting for spin-polarized STM imaging as first pointed out in Ref. 13. Although this particular study was not aimed at achieving spin-polarized contrast, we employed $\mathrm{MnNi}$ tips, as in our experience they also more readily yield conventional atomic resolution images. The results of the spinpolarized contrast studies on the $\mathrm{Fe}_{3} \mathrm{O}_{4}(111)$ will be published in Ref. 14. Details of the scanning conditions are presented with reference to the relevant figures.

We have employed three in situ sample preparation procedures. The initial step of all the three procedures consists of annealing the sample in UHV (base pressure $10^{-11} \mathrm{mbar}$ ) to a temperature of about $1000 \mathrm{~K}$. This treatment has a significant impact on the surface stoichiometry, resulting in a reduction of the $\mathrm{O} / \mathrm{Fe}$ ratio as observed by AES. The $\mathrm{O}_{(510 \mathrm{eV})} / \mathrm{Fe}_{(703 \mathrm{eV})}$ peak ratio drops to a value of 1.1 after anneal (compared with 1.33 for the stoichiometric surface). Similar results have been recorded for the $(100) \mathrm{Fe}_{3} \mathrm{O}_{4}$ 

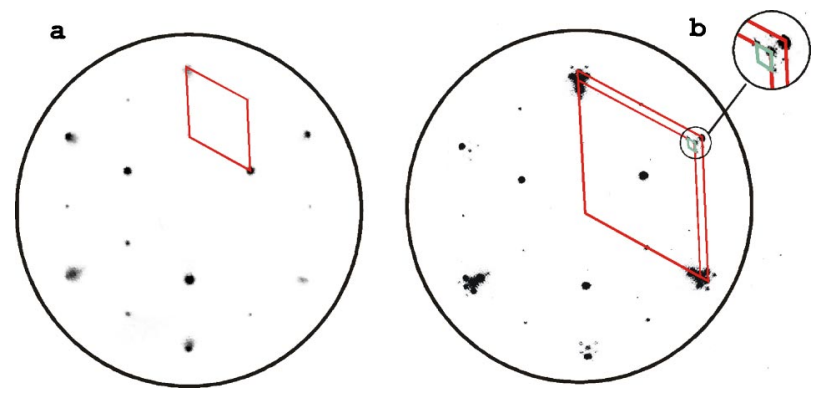

FIG. 2. (Color online) LEED patterns of $\mathrm{Fe}_{3} \mathrm{O}_{4}(111)$ surface at $E=67 \mathrm{eV}$ : (a) tetrahedral site Fe termination, $6.0 \pm 0.3 \AA$ periodicity indicated, and (b) $\mathrm{Fe}_{3} \mathrm{O}_{4}(111)$ overoxidized surface. Two large rhombuses indicate the periodicities of $3.1 \pm 0.1 \AA$ and $2.8 \pm 0.1 \AA$; the small rhombus in the insert shows a $42 \pm 3 \AA$ superstructure.

surface. ${ }^{8}$ The final anneal step aimed at recovering the subsurface stoichiometry was different for the three in situ preparation procedures. Depending on the details of the final anneal step, this leads to the formation of the so-called "regular" termination, "oxygen" termination, or the "intermediate" case. These are described below in detail along with the conditions of the final anneal step.

\section{A. "Regular" termination}

This termination was formed as a result of annealing in an oxygen atmosphere of $10^{-6} \mathrm{mbar}$ at $950 \mathrm{~K}$ for $15 \mathrm{~min}$. The oxygen was then promptly pumped out of the chamber and the sample was cooled down to room temperature in UHV conditions. Following this sample treatment, AES measurements indicate an $\mathrm{O}_{(510 \mathrm{eV})} / \mathrm{Fe}_{(703 \mathrm{eV})}$ ratio of about 1.3, which is consistent with the value for the magnetite bulk. ${ }^{8}$ The LEED analysis of the surface after such annealing yielded patterns with hexagonal symmetry and a periodicity of $6 \AA$, which is consistent with that of the $\mathrm{Fe}_{3} \mathrm{O}_{4}$ bulk termination [Fig. 2(a)].

The $\mathrm{Fe}_{3} \mathrm{O}_{4}$ (111) Fe-tetrahedral termination is referred to here as "regular," as it is commonly reported for the magnetite [111] surface. ${ }^{5,16,17}$ Figure 3 shows a typical STM image

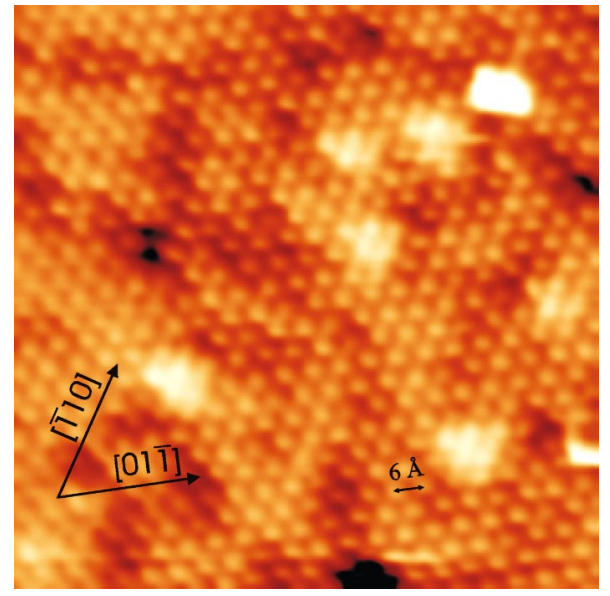

FIG. 3. (Color online) $15 \times 15 \mathrm{~nm}^{2} \mathrm{STM}$ image of $\mathrm{Fe}_{3} \mathrm{O}_{4}(111)$ showing the regular type of termination with $\mathrm{Fe}$ ions in tetrahedral sites with $6.0 \pm 0.3 \AA$ periodicity $\quad\left(V_{\text {bias }}=0.3 \mathrm{~V}, I_{\mathrm{t}}=0.1 \mathrm{nA}\right.$, MnNi tip). of the regular termination. It represents a $p(1 \times 1)$ hexagonal lattice with a $6 \AA$ periodicity. The hexagonal pattern of bright spots corresponds to $\mathrm{Fe}^{3+}$ atoms in tetrahedral positions, and therefore the "regular" termination can be considered as one of the six possible bulk terminations [Fig. 1(c)]. A height difference of about $4.8 \AA$ between terraces was observed by us on this surface, which is also consistent with the model of the tetrahedrally coordinated Fe-cation termination [Fig. 1(a)].

\section{B. "Oxygen" termination}

The second in situ preparation procedure was performed with the aim of obtaining an oxygen-rich surface of magnetite (111). According to the phase diagram of the Fe-O system, ${ }^{17}$ iron oxide that is annealed to equilibrium at an oxygen pressure of $10^{-6}$ mbar at a temperature above $900 \mathrm{~K}$ is magnetite. Oxygen-rich iron oxide phases, like $\alpha-\mathrm{Fe}_{2} \mathrm{O}_{3}$, start to form at the same oxygen pressure if the annealing temperature is reduced, and the $\mathrm{FeO}$ phase is formed at much higher temperature or lower oxygen pressure, respectively. Therefore, in order to obtain the oxygen-rich surface we have chosen a second in situ preparation procedure consisting of annealing the sample at $950 \mathrm{~K}$ in an oxygen partial pressure of $10^{-6}$ mbar with subsequent cooling to room temperature at this oxygen pressure. We anticipated that this procedure should significantly change the oxygen concentration on the surface. However, we expected that the stoichiometry change would only affect a shallow subsurface layer of the crystal due to the relatively short cooling time.

Auger measurements indicate that following such an anneal, the $\mathrm{O}_{(510 \mathrm{eV})} / \mathrm{Fe}_{(703 \mathrm{eV})}$ ratio increases to 1.45 . The LEED pattern displays fractional order spots around the integral-order spots, due to a well-defined hexagonal superlattice with a periodicity of $42 \pm 3 \AA$ [Fig. 2(b)]. Figure 4(a) shows an STM image of the oxygen-terminated surface formed after the second in situ preparation procedure. The surface is covered by superperiodic structures with a $42 \AA$ periodicity, in agreement with the LEED diffraction pattern. These superstructures are highly regular and cover virtually the entire sample surface. Higher resolution STM images [Fig. 4(b)] show the atomic arrangement within these superperiodic structures. The pattern is made by the repetition of three distinct areas, marked as areas I, II, and III in Fig. 4(b). Detailed analysis shows that area I has a periodicity of $3.1 \pm 0.1 \AA$, while areas II and III both have a periodicity of $2.8 \pm 0.1 \AA$ along the [01-1] direction. The atomic arrangements in areas II and III have exactly the same symmetry and periodicity. The LEED pattern is well in line with the STM results. The separation between the satellite spots and the integral-order spots is consistent with a $42 \AA$ superperiodicity, whereas the positions of the doubled main spots are consistent with the presence of two periodicities: 2.8 and $3.1 \AA$ [Fig. 2(b)].

Superstructures formed on the $\mathrm{Fe}_{3} \mathrm{O}_{4}$ (111) surface were reported previously in Refs. 15 and 16 and interpreted as a biphase structure consisting of two phases: $\mathrm{FeO}$ and $\mathrm{Fe}_{3} \mathrm{O}_{4}$, arranged in a superlattice. This interpretation cannot be applied to our results for a number of reasons. First, the LEED 


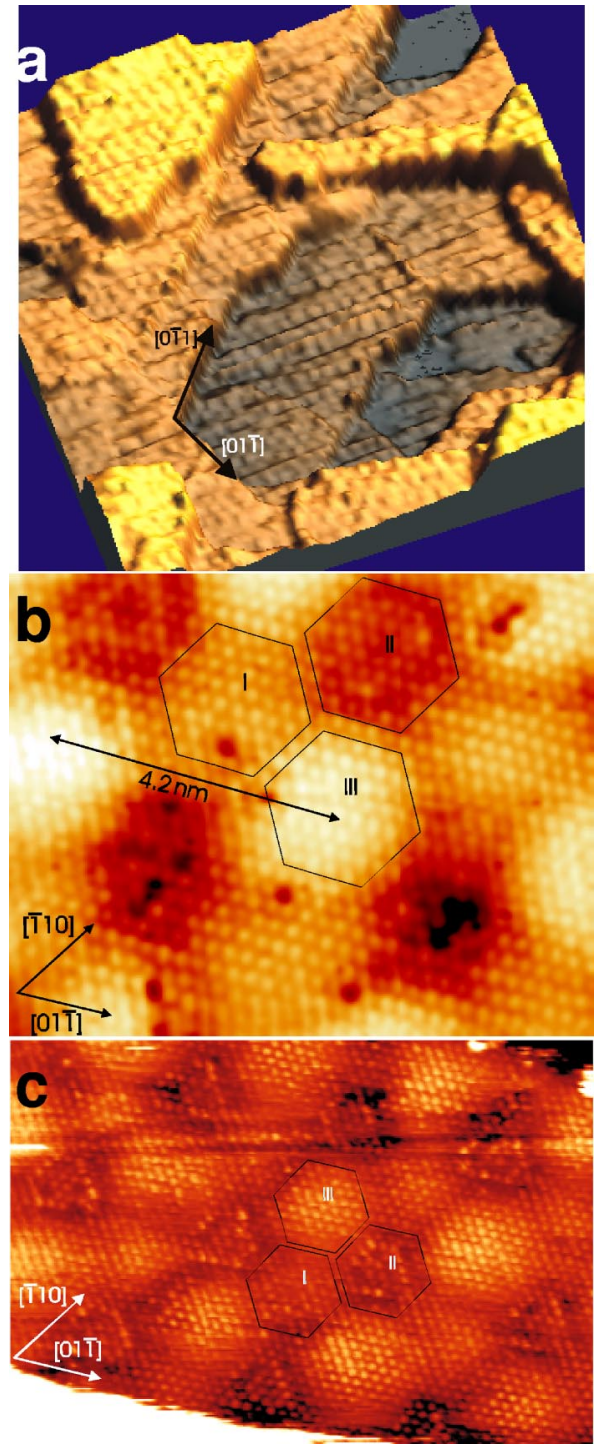

FIG. 4. (Color online) (a) $120 \times 120 \mathrm{~nm}^{2}$ STM image of overoxidized $\mathrm{Fe}_{3} \mathrm{O}_{4}(111)$ surface representing large terraces covered by superstructures of about $42 \AA$ period; (b) $10 \times 8 \mathrm{~nm}^{2}$ STM image of superperiodic pattern seen in (a). Area I has 3.1 $\pm 0.1 \AA$ interatomic periodicity, and areas II and III have $2.8 \pm 0.1 \AA$ periodicity ( $V_{\text {bias }}$ $=-1.0 \mathrm{~V}, I_{\mathrm{t}}=0.1 \mathrm{nA}, \mathrm{MnNi}$ tip). (c) $15 \times 10 \mathrm{~nm}^{2} \mathrm{STM}$ image of the same sample as in (a), with different bias: $V_{\text {bias }}$ $=-0.5 \mathrm{~V}$. The height difference between areas II and III now is $0.4 \AA$, instead of $0.6 \AA$ as in (b) $\left(V_{\text {bias }}=-0.5 \mathrm{~V}, I_{\mathrm{t}}=0.1 \mathrm{nA}\right.$, MnNi tip).

pattern [Fig. 2(b)] does not suggest formation of another iron oxide phase like FeO (the LEED pattern should show floreting about the first and second zone spots in the case of $\mathrm{FeO}$ and $\mathrm{Fe}_{3} \mathrm{O}_{4}$ phases simultaneously present on the surface ${ }^{11}$ ). Second, we expect that following our in situ preparation procedure the surface is oxidized rather than reduced, and this is indeed confirmed by the increase in the O/Fe AES ratio. Therefore, the formation of a reduced iron oxide, $\mathrm{FeO}$, is not expected in our experiment. Concerning the possibility of forming higher oxidation iron oxide phase on magnetite surface, we should note here that the structural difference between $\mathrm{Fe}_{3} \mathrm{O}_{4}$ and $\mathrm{Fe}_{2} \mathrm{O}_{3}$ (Ref. 1) makes it easy to distinguish on the LEED pattern. Similar superstructure formed on $\alpha$ $-\mathrm{Fe}_{2} \mathrm{O}_{3}$ surface was reported in Ref. 16 and explained as a biphase structure of $\alpha-\mathrm{Fe}_{2} \mathrm{O}_{3}(0001)$ and $\mathrm{FeO}$ (111). Comparing the LEED pattern in Fig. 2 and in Ref. 18, we could find no indications of the $\mathrm{Fe}_{2} \mathrm{O}_{3}(0001)$ phase on the surface in our case. In addition, we should note that the multiplephase structures often lead to formation of antiphase boundaries. That has not been observed in our case.

It would be in line with our sample preparation procedure and Auger results to suggest, that the 3.1 and $2.8 \AA$ periodicities of the areas I, II, and III in the oxygen termination result from a closed-packed oxygen layer formed on the magnetite surface. There are additional experimental evidences in favor of the oxygen-termination model. First, in the oxygen termination, the atomically resolved STM images could be obtained much more readily at a negative sample bias, suggesting that we likely image the filled states of electronegative surface ions. Second, the smallest separation step between terraces is $2.5 \pm 0.6 \AA$, which is consistent with the distance between two nearest-neighbor oxygen layers in the magnetite [111] bulk structure.

Another interesting property of the observed superstructure is that the boundaries between the areas I, II, and III shift significantly depending on the bias voltage applied to the sample, resulting in changes of the shape of the superstructure. This is shown in Fig. 4(c). It is interesting to note that the shape of the superstructure was also altered when different tips (W, Ni, MnNi) were used. The superstructure periodicity does not depend on the bias and the tip material used. However, we should point out that the height difference between the areas I, II, and III does depend on the tunnel bias. These facts evidently indicate that the superstructure depicts an electronic rather than a structural effect.

We were able to reproducibly toggle between the "regular" and "oxygen" terminations. For example, we could readily switch the surface to the regular termination by subjecting the sample with oxygen termination to a 15-min anneal as described above. Alternatively, we could remove the subsurface layer by $\mathrm{Ar}^{+}$ion sputtering $(10 \mathrm{~min}, 1 \mathrm{keV})$ and again form the oxygen termination with the superstructure by repeating the oxidation procedure.

The oxygen terminated magnetite surface was found to be very stable. Two days after the sample preparation, we were still able to achieve atomic resolution STM on the oxygenterminated surface provided the sample was kept under UHV during that time.

\section{C. "Intermediate" case}

In order to better understand the formation of the oxygen termination and, in particular, the superstructure within it, we reduced the temperature of the final anneal step in oxygen to $850 \mathrm{~K}$. The aim was to form a partially closed-packed oxygen layer on the surface and establish how this affects the formation of the superstructure. Following this anneal, the LEED pattern of the surface was similar to the one of the oxygen termination [Fig. 2(b)], but with weaker satellite spots. Figure 5 shows the STM images corresponding to this altered sample preparation. Three types of terraces are seen 

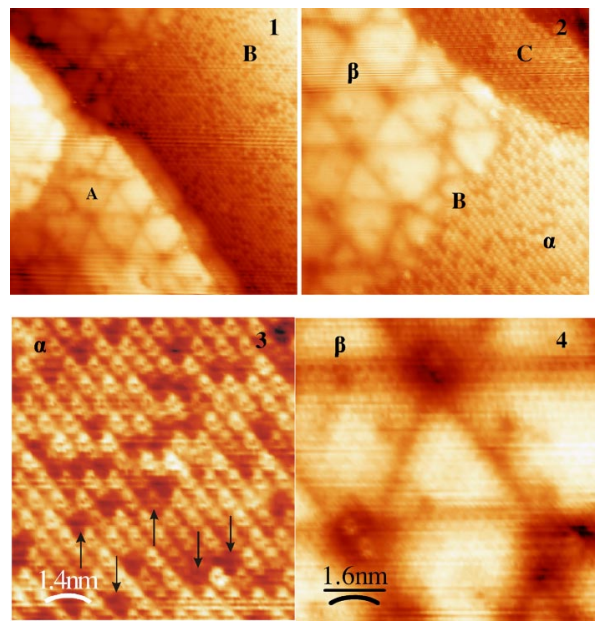

FIG. 5. (Color online) $\mathrm{Fe}_{3} \mathrm{O}_{4}(111)$ surface after annealing in oxygen at $850 \mathrm{~K}: 30 \times 30 \mathrm{~nm}^{2}(1)$ and $20 \times 20 \mathrm{~nm}^{2}$ (2) STM images showing few terraces with different atomic arrangement: terrace $(\mathbf{A})$ completely covered by superstructure; terrace $(\mathbf{C})$ represents region of $6 \AA$ periodicity; terrace $(\mathbf{B})$ has a mixture of atomic arrangements presented on terraces $\mathbf{A}$ and $\mathbf{C}$; (3) zoom-in on region $\boldsymbol{\alpha}, 6 \AA$ periodicity region showing the splitting to $3 \AA$ arrangement (arrows indicate the oxygen defects); (4) zoom-in of superstructure region $\boldsymbol{\beta}\left(V_{\text {bias }}=-1.0 \mathrm{~V}, I_{\mathrm{t}}=0.1 \mathrm{nA}, \mathrm{Ni}\right.$ tip $)$.

in Fig. 5: terrace $\mathbf{A}$ is covered by a superstructure similar to the one described above (oxygen termination); terrace $\mathbf{C}$ exhibits a hexagonal lattice with a $6 \AA$ periodicity, while another terrace $\mathbf{B}$ represents the combination of the above two structures. The hexagonal lattice with $6 \AA$ periodicity, however, is not equivalent to the regular termination. A zoom into the area $\alpha$ of terrace $\mathbf{B}$ is shown in Fig. 5(3). Most of the $6 \AA$ protrusions split into three atoms positioned with a local triangular geometry and a periodicity of $3 \AA$. Many protrusions contain only two atoms and therefore correspond to the locations of defects where the oxygen atoms are missing. Some of the latter are indicated with arrows [Fig. 5(3)]. We suggest that terrace $\mathbf{C}$ and the $\boldsymbol{\alpha}$ region of terrace $\mathbf{B}$ consist of an incomplete layer of oxygen atoms and are different from the oxygen termination described above in that the layer is not complete. The separation between the terraces $(2.5 \pm 0.6 \AA)$ is essentially identical to the one observed in the case of oxygen-terminated planes.

The results explained in this section provide further support to our interpretation of the $42 \AA$ superstructure being a long-range electronic effect triggered by the change of the $\mathrm{O} / \mathrm{Fe}$ ratio on the surface. A representative case is shown in Fig. 5(2). The upper part ( $\boldsymbol{\beta})$ of the terrace $\mathbf{B}$ is a segment having a completed oxygen termination, i.e., having a very small number of defects. Such areas invariably form longrange order identical to the one described above. A zoom-in of the region $\boldsymbol{\beta}$ is shown in Fig. 5(4). If the oxygen layer contains defects and therefore is incomplete (region $\boldsymbol{\alpha}$ ), the long-range order is not formed. From our STM results we could estimate that the critical defect density that destroys formation of the long-range order at room temperature is about $10 \%-20 \%$. One could envisage that the long-range order may still be formed on such surfaces with defects at a lower temperature. In other words, the temperature of the long-range order transition is a function of the density of defects.

The $42 \AA$ periodicity of the superstructure was not maintained in the vicinity of the terrace edges or in the vicinity of the boundaries between areas with the complete oxygen termination and termination with defects [Fig. 5(2)].

\section{MODEL OF THE SUPERSTRUCTURE FORMATION}

Our model relies on the existence of a tensile strain (lattice deformation) at the oxygen-terminated surface. The presence of this lattice deformation is evident from our STM data. To further validate the notion of such deformation, we have performed density functional theory (DFT) calculations for the structure of the (111) surface of magnetite. The CASTEP algorithm within the local density approximation of DFT $^{19}$ was used to calculate total energies and optimize the atomic positions on the surface to minimize the energy. A good example demonstrating a successful corroboration between STM experiment on transition metal oxides and DFT calculations was provided in a recent publication. ${ }^{20}$

As explained above, the surface with $3 \AA$ periodicity could represent two possible types of closed-packed oxygen layer termination located either above a layer of $B$-site $\mathrm{Fe}^{2+, 3+}$ cations, or an $\mathrm{Fe}$ multilayer with a mixture of $A$ - and $B$-sites cations (Fig. 1). The total charge density distribution calculated for both types of oxygen terminations (Fig. 6) shows the local charge density maxima above the oxygen sites, which is in agreement with our STM data. We were not able to run a geometry optimization calculation for an extended slab to find out the condition of the superstruc-
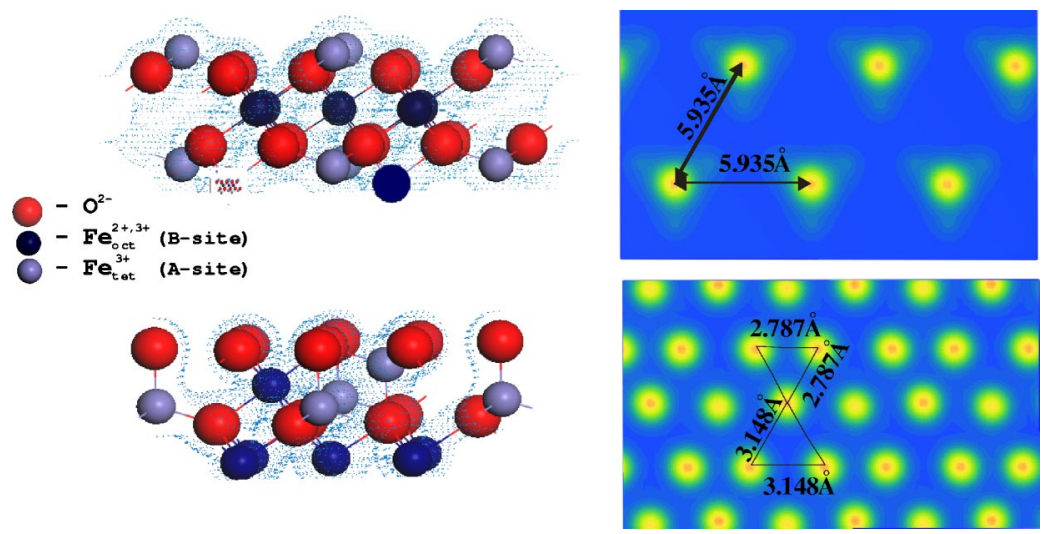

FIG. 6. (Color online) $\mathrm{Fe}_{3} \mathrm{O}_{4}(111)$ crystal structure: Fe-tetrahedral termination (left-top); oxygen termination (left-bottom) with the 0.2 level of electron density shown as isosurface; 2D map of the total charge density (right) above surface calculated for the vacuum slabs shown on the left. Hexagonal symmetry of $3 \AA$ oxygen lattice is distorted by strain as shown by triangles marking atom positions. DFT calculations were performed for one unit cell slab. 
ture formation on (111) surface. Nevertheless, the calculation for one unit cell slab indicates that the positions of some oxygen atoms shifted laterally (Fig. 6) with respect to their bulk positions, and therefore point to the formation of strain along the surface of the oxygen termination. The mechanism of such strain is clear: dangling $\mathrm{O}$ bonds at the surface result in excess electrons that are retained in the topmost oxygen layer. The resulting Coulomb repulsion established between the oxygen anions containing these excess electrons produces an increase in interionic separation from 2.8 to $3.1 \AA$.

As we pointed out above, the superstructure represents an electronic rather than simply a structural effect, meaning that there is a significant difference in tunneling conductance in areas I-III. There are two types of instabilities in crystals that link together excessive strain and changes in electronic properties and create a long-range order: localized polaron and charge density waves (CDW). The concept of polarons has been applied to magnetite in the past. For example, it was suggested in Ref. 21 that at room temperature the conductivity of magnetite could be explained in terms of polaronic transport. Particularly, the concept of Jahn-Teller polarons as local distortions of the lattice around $\mathrm{Fe}^{3+}$ ions was proposed in Refs. 21 and 22. Another possibility is to base the model on the concept of a CDW. The CDW is also an electronically induced lattice instability accompanied by a modulation of the electronic density. In the case of one-dimensional systems, a CDW is known to occur at a wave vector $q=2 k_{\mathrm{F}}$, and is known as Peierls instability. In the case of $2 \mathrm{D}$ and $3 \mathrm{D}$ systems, a CDW can develop for Fermi liquids with a nested Fermi surface..$^{23}$ The surface structure of magnetite could be particularly susceptible to modulation of the electronic structure due to the presence of $\mathrm{Fe}^{2+}$ and $\mathrm{Fe}^{3+}$ ions at the $B$ sites. The strain could cause reallocation of charges between the octahedral $B$ sites. Reallocation of charges between $A$ - and $B$-sites also could be considered.

To complete the model, further experiments are needed to explore electronic and magnetic properties of the reported superstructure.

\section{SUMMARY}

Three different terminations on the (111) surface of a synthetic $\mathrm{Fe}_{3} \mathrm{O}_{4}$ single crystal were formed and studied using
LEED, AES, and STM with a range of different nonmagnetic, ferromagnetic, and antiferromagnetic tip materials. The three sample preparation procedures leading to the three terminations varied only in the conditions of the last step: in situ anneal in oxygen. They result in three different values of the surface stoichiometry. The three surface terminations were identified as a termination of $\mathrm{Fe}$ cations in tetrahedral sites called the regular termination, complete oxygen layer termination called the oxygen termination, and the intermediate case of an oxygen layer termination containing defects.

The oxygen-terminated surface forms a hexagonal superstrucure with periodicity of $42 \AA$. In the superstructure, the atomic level periodicities are virtually unaffected except for the modulation of strain that could be observed using STM and LEED. The change of the superstructure shape depends on the bias voltage. It is also sensitive to the type of tip material used in the STM. We conclude that the superstructure is an electronic effect resulting from an electron-lattice instability, rather than a mosaic of different iron oxide phases. The superstructure is sensitive to the oxygen to iron ratio at the surface. Once a nearly complete layer of oxygen covers the surface, lateral strain is developed, triggering the formation of the superstructure. On the other hand, if the layer contains defects in the form of missing oxygen atoms, the strain is reduced and the superstructure disappears. From our STM data we could work out that the critical density of defects destroying the formation of the superstructure at room temperature is approximately $10 \%-20 \%$ of the complete oxygen-terminated layer. We have performed DFT calculations for the regular and two different oxygen terminations. The results of the calculations suggest that unlike in the regular termination, each of the two possible oxygen terminations results in a significant lateral strain that may lead to the formation of long-range order through electron-lattice instability. We suggest either polaronic or CDW electronlattice instability may develop the superstructure on the surface of magnetite.

\section{ACKNOWLEDGMENT}

This work was supported by Science Foundation of Ireland (SFI) under Contract 00/PI.1/C042.
${ }^{1} \mathrm{R}$. Cornell and U. Schwertmann, The Iron Oxides (Verlagsgellschaft mbH, Cambridge, 1996).

${ }^{2}$ H. Seo, M. Ogata, and H. Fukuyama, Phys. Rev. B 65, 085107 (2002).

${ }^{3}$ J. Ahdjoudj, C. Martinsky, C. Minot, M. A. Van Hove, and G. A. Somorjai, Surf. Sci. 443, 133 (1999).

${ }^{4}$ A. R. Lennie, N. G. Condon, F. M. Leibsle, G. Murry, P. W. Thornton, and D. J. Vaughan, Phys. Rev. B 53, 10244 (1996).

${ }^{5}$ M. Ritter and W. Weiss, Surf. Sci. 432, 81 (1999).

${ }^{6}$ K. T. Rim, J. P. Fitts, T. Muller, K. Adib, N. Camillone, R. M. Osgood, S. A. Joyce, and G. W. Flynn, Surf. Sci. 541, 59 (2003).
${ }^{7}$ M. L. Rudee, D. J. Smith, and D. T. Margulies, Phys. Rev. B 59, R11 633 (1999).

${ }^{8}$ G. Mariotto, S. Murphy, and I. V. Shvets, Phys. Rev. B 66, 245426 (2003).

${ }^{9}$ R. Wiesendanger, I. V. Shvets, D. Burgler, G. Tarrach, H. J. Guntherodt, J. M. D. Coey, and S. Graser, Science 255, 583 (1992).

${ }^{10}$ G. Mariotto, M. D. D’Angelo, and I. V. Shvets, Rev. Sci. Instrum. 70, 3651 (1999).

${ }^{11}$ R. Koltun, M. Herrmann, V. Guentherodt, and V. A. M. Brabers, Appl. Phys. A: Mater. Sci. Process. A73, 49 (2001).

${ }^{12}$ S. F. Ceballos, G. Mariotto, S. Murphy, and I. V. Shvets, Surf. 
Sci. 523, 131 (2003).

${ }^{13}$ A. A. Minakov and I. V. Shvets, Surf. Sci. 236, L377 (1990).

${ }^{14}$ N. Berdunov, M. Murphy, G. Mariotto, and I. V. Shvets, J. Appl. Phys. 95, 6891 (2004).

${ }^{15}$ N. G. Condon, F. M. Leibsle, T. Parker, A. R. Lennie, D. J. Vaughan, and G. Thornton, Phys. Rev. B 55, 15885 (1997).

${ }^{16}$ S. K. Shaikhutdinov, M. Ritter, X. G. Wang, H. Over, and W. Weiss, Phys. Rev. B 60, 11062 (1999).

${ }^{17}$ W. Weiss and W. Ranke, Prog. Surf. Sci. 70, 1 (2002).

${ }^{18}$ N. G. Condon, F. M. Leibsle, A. R. Lennie, P. W. Murray, D. J.
Vaughan, and G. Thornton, Phys. Rev. Lett. 75, 1961 (1995).

${ }^{19}$ M. D. Segall, P. J. D. Lindan, M. J. Probert, C. J. Pickard, P. J. Hasnip, S. J. Clark, and M. C. Payne, J. Phys.: Condens. Matter 14, 2717 (2002).

${ }^{20}$ Sh. K. Shaikhutdinov, R. Meyer, D. Lahav, M. Baumer, T. Kluner, and H.-J. Freund, Phys. Rev. Lett. 91, 076102 (2003).

${ }^{21}$ J. B. Sokoloff, Phys. Rev. B 5, 4496 (1972).

${ }^{22}$ L. V. Gasparov, D. B. Tanner, D. B. Romero, H. Berger, G. Margaritondo, and L. Forro, Phys. Rev. B 62, 7939 (2000).

${ }^{23}$ G. Grunder, Rev. Mod. Phys. 60, 1129 (1988). 